\title{
Potentiel en biogaz des résidus agropastoraux et des excréments humains du bassin versant du fleuve Sassandra (Côte d'Ivoire)
}

\author{
Lacina COULIBALY ${ }^{1}$, Jean-Marie Pétémanagnan OUATTARA ${ }^{1 *}$ et Seydou TIHO ${ }^{2}$ \\ ${ }^{1}$ Laboratoire d'Environnement et de Biologie Aquatique, Unité de Formation et de Recherche en Sciences et \\ Gestion de l'Environnement (UFR-SGE), Université d'Abobo-Adjamé, 02 BP 801 Abidjan 02, Côte d'Ivoire. \\ ${ }^{2}$ Laboratoire de Cytologie et Biologie Animales, Unité de Formation et de Recherche en Sciences de la Nature \\ (UFR-SN), Université d'Abobo-Adjamé, 02 BP 801 Abidjan 02, Côte d'Ivoire. \\ *Auteur correspondant : E-mail: jm_petemanagnan@yahoo.fr ; coulacina2003@yahoo.fr
}

\section{RESUME}

La potentialité en biogaz des excréments humains et des résidus agropastoraux du bassin versant du fleuve Sassandra (BVS) a été évaluée à partir des statistiques agricoles et de la population, ainsi que des indices de productivité de biogaz. Egalement, la mise en œuvre des technologies de production de biogaz dans ledit bassin a été analysée. Le BVS renferme un potentiel énergétique total de $604,2 \times 10^{6} \mathrm{~m}^{3}$ de biogaz. Ce potentiel est supérieur aux besoins énergétiques annuels du monde rural en Côte d'Ivoire (1 $800 \mathrm{GWh})$. Cependant, celui-ci est inégalement réparti entre les localités. En considérant les différents résidus, la contribution des résidus agricoles $\left(541 \times 10^{6} \mathrm{~m}^{3}\right)$ est plus importante par rapport à celle des déchets d'élevages $\left(45,7 \times 10^{6} \mathrm{~m}^{3}\right)$ et celle des excréments humains $\left(17,5 \times 10^{6} \mathrm{~m}^{3}\right)$. La complexité de gestion des digesteurs utilisant des résidus agricoles solides ne permet pas de les conseiller pour les productions domestiques de biogaz. Toutefois, des unités industrielles peuvent être installées à Soubré, Daloa et Issia où des quantités élevées de résidus agricoles sont générées. Par ailleurs, de petites unités décentralisées de production de biogaz peuvent être installées dans les petites localités pour exploiter les résidus d'élevages.

(C) 2012 International Formulae Group. All rights reserved.

Mots clés: Agriculture, biogaz, élevage, production, résidus, Sassandra.

\section{INTRODUCTION}

La question de la disponibilité de l'énergie remonte aux origines de l'homme. Il n'est pas utopique de nos jours de dire que le développement s'arrête là où les ressources en énergie se font rares. Car l'énergie permet l'accès à l'éducation, améliore les conditions de vie des populations et permet de faire des activités lucratives ; ce qui contribue à réduire la pauvreté. D'ailleurs, l'accès à l'énergie, en tant que bien essentiel, était au centre des débats du sommet international sur le développement durable à Johannesburg en 2002 où le lien entre énergie et pauvreté a été mis en évidence (IEA, 2002). La crise énergétique de ces dernières décennies, caractérisée par la flambée des prix de l'énergie fossile, témoigne de la nécessité de rechercher des énergies alternatives (Chynowheth et al., 2001 ; Wauthelet, 2001).

La biomasse demeure la principale source d'énergie de 2,4 milliard de population dans les pays en voie de développement (IEA, 1998). En effet, la biomasse contribue à plus 
de $50 \%$ dans la consommation totale d'énergie en milieu rural. En Afrique, cette contribution avoisine $90 \%$ des énergies consommées, dont les deux tiers satisfont des besoins domestiques (FAO, 1998). Tel est le cas en Côte d'Ivoire où la principale source d'énergie des ménages demeure la biomasse (97\%) (IEA, 2000 ; PNUD, 2003 ; ESMAP, 2005). L'utilisation de cette source d'énergie pose des problèmes environnementaux majeurs (dégradation des terres, déforestation, avancé du désert, réduction des puits de $\mathrm{CO}_{2}$ ), de santé (maladies pulmonaires et d'yeux), ainsi qu'une occupation abusive des femmes (91\% du temps) (Mehretu et Mutambira, 1992 ; OMS, 2000). En outre, la disponibilité $\mathrm{du}$ bois devient rare dans certaines zones en raison de leur surexploitation; ce qui oblige les femmes et les enfants à couvrir de longues distances pour la collecte. Le biogaz est une source d'énergie dont la production se fait à partir de divers résidus organiques (excréments humains, résidus agropastoraux, etc.) (Cho et al., 1995 ; Gowga et al., 1995 ; Kivaisi et Rubindamayugi, 1996; Zhang et Zhang, 1999 ; Moller et al., 2004 ; Nabinta et al., 2007). Cette énergie est flexible et fiable, et des unités de production peuvent être réalisées de manière décentralisée dans le milieu rural (Chanakya et al., 2004). Cependant, sa mise en œuvre dans les pays en développement telle que la Côte d'Ivoire est encore modeste. Cette situation est due a l'inexistence de politique de dissémination du biogaz, laquelle commence par une évaluation rigoureuse des substrats à utiliser (FAO, 1998 ; Kumar et al., 2002 ; Nelson et al., 2004). La Côte d'Ivoire étant un pays à économie agricole, elle produit une masse importante de résidus agropastoraux. Ces résidus pourraient être valorisés pour la production de biogaz; ce qui permettrait de réduire les besoins énergétiques des populations et améliorer leur condition de vie (PNUD, 2001 ; Chanakya et al., 2004), ainsi que de ralentir la déforestation et les émissions de gaz à effet de serre. En effet, l'utilisation d'un digesteur de $4 \mathrm{~m}^{3}$ de biogaz permet de protéger annuellement la destruction de 37 ha de forêt (PNUD, 2001). Par ailleurs, la digestion des excréments humains permettrait de détruire les pathogènes (Billaud et Varagnat, 1983) et de mieux contrôler les épisodes diarrhéiques dans le milieu rural.

L'objectif de ce travail est (i) de quantifier le volume de biogaz susceptible d'être produit dans les différentes localités du bassin versant du fleuve Sassandra à partir des résidus agropastoraux et des excréments humains et (ii) de discuter la mise en œuvre d'unités de production de biogaz dans ledit bassin versant.

\section{MATERIEL ET METHODES}

\section{Zone d'étude}

Le bassin versant du fleuve Sassandra (BVS) est situé à l'Ouest de la Côte d'Ivoire (Figure 1). Le fleuve Sassandra qui le caractérise est long de $650 \mathrm{~km}$ et prend sa source dans la région de Beyala en Guinée (Girardet al., 1971). La superficie de ce bassin versant est de $75000 \mathrm{~km}^{2}$. Il compte environ 4 276483 habitants (INS, 2000), soit un quart de la population de Côte d'Ivoire, dont $60 \%$ vivent en milieu rural.Cette population est fortement concentrée dans les chefs lieu de région (Daloa, Gagnoa, Guiglo, Man, Touba) et de département (Bangolo, Biankouma, Duékoué, Issia, Sassandra, Sinfra, Soubré, Vavoua). Elle exerce une pression importante sur les forêts en vue de pratiquer l'agriculture et de rechercher de la biomasse énergie.

\section{Données}

Les données sur les résidus agricoles et d'élevages proviennent des statistiques agricoles et d'élevage de 2005 de la Direction des statistiques de la documentation et de l'informatique du Ministère de l'agriculture de Côte d'Ivoire. Concernant la population, les statistiques de 2000 de l'Institut National des Statistiques (INS) ont été utilisées.

Les statistiques relatives à l'élevage de la volaille dans le BVS n'ont pas pu être obtenues. Cependant, l'absence de ces 
données n'influence pas significativement les résultats à cause de sa faible proportion (FAO, 2005).

\section{Evaluation du potentiel en biogaz des résidus \\ Cette évaluation a consisté à} déterminer les quantités de résidus générées par la population, la production agricole et l'élevage. Ensuite, les volumes de biogaz équivalents pour chaque type de résidus ont été estimés à l'aide d'indices de productivité.

\section{Excréments humains}

Le potentiel de production de biogaz des excréments humains $\left(\mathrm{P}_{\mathrm{Hu}}\right)$ de chaque localité a été calculé par la relation (1) :

$\mathrm{P}_{\mathrm{Hu}}\left(\mathrm{m}^{3}\right)=\mathrm{POP}_{\mathrm{Hu}} \times \mathrm{RJ}_{\mathrm{Hu}} \times \mathrm{Ip} \times 365$

$\mathrm{POP}_{\mathrm{Hu}}$ : Effectif de la population locale,

$\mathrm{RJ}_{\mathrm{Hu}}$ : Production journalière d'excréments (kg/personne),

Ip : Indice de productivité,

365 : Nombre de jours dans l'année.

Les valeurs $0,4 \mathrm{~kg} /$ personne et 0,028 $\mathrm{m}^{3} /\left(\mathrm{kg}\right.$ excréments), respectivement pour $\mathrm{RJ}_{\mathrm{Hu}}$ et Ip ont été utilisées pour les calculs (Nagamani et Ramasamy, 2005).

\section{Déjections animales}

Le potentiel de production de biogaz des déjections de chaque type d'élevage $\left(\mathrm{P}_{\mathrm{An}}\right)$ a été inventorié par la relation (2) :

$\mathrm{P}_{\mathrm{An}}\left(\mathrm{m}^{3}\right)=\mathrm{POP}_{\text {An }} \times \mathrm{QMO} \times \mathrm{Ip} \times 365$

$\mathrm{POP}_{\text {An }}$ : Population animale du type d'élevage considéré,

QMO: Quantité journalière de matière organique (kg/tête),

Ip : Indice de productivité,

365 : Nombre de jours dans l'année.

Les valeurs de QMO et Ip des différents animaux sont consignées dans le Tableau 1.

\section{Résidus agricole}

Le potentiel de production annuelle de biogaz des résidus agricoles a été estimé à partir de la quantité de matières sèches (QMS) desdits résidus. Cette quantité de matière sèche a été calculée à partir de la relation (3) :

$\mathrm{QMS}=\mathrm{m} \times$ Cres $\times \mathrm{Cms}$

Le potentiel de biogaz des résidus solides agricoles $\left(\mathrm{P}_{\mathrm{Ag}}\right)$ a été évalué à partir d'équation (4).

$\mathrm{P}_{\mathrm{Ag}}=\mathrm{QMS} \times \mathrm{Ip}$

Les relations (3) et (4) combinées donnent la relation (5):

$\mathrm{P}_{\mathrm{Ag}}=(\mathrm{m} \times$ Cres $\times \mathrm{Cms}) \times \mathrm{Ip}$

$\mathrm{m}$ : Masse de la production de la culture considérée (t),

Cres : Coefficient exprimant la quantité de résidus générés en fonction de la production agricole,

Cms : proportion de matières sèches contenues dans les résidus,

Ip : indice de productivité.

Les valeurs de Cms, Cres et Ip utilisées sont consignées dans le Tableau 2.

\section{Pouvoir calorifique}

Le pouvoir calorifique inférieur du biogaz ayant un pourcentage de méthane compris entre 50 et $70 \%$ varie entre 4,71 et $6,59 \mathrm{Kwh} / \mathrm{m}^{3}$.

\section{Détermination du mode d'utilisation des résidus agricoles}

Elle a été effectuée par une recherche documentaire sur l'utilisation des différents résidus agricoles dans le BVS (compostage, biométhanisation, incinération in situ, combustion pour les besoins domestiques, transformation en charbon de bois). 


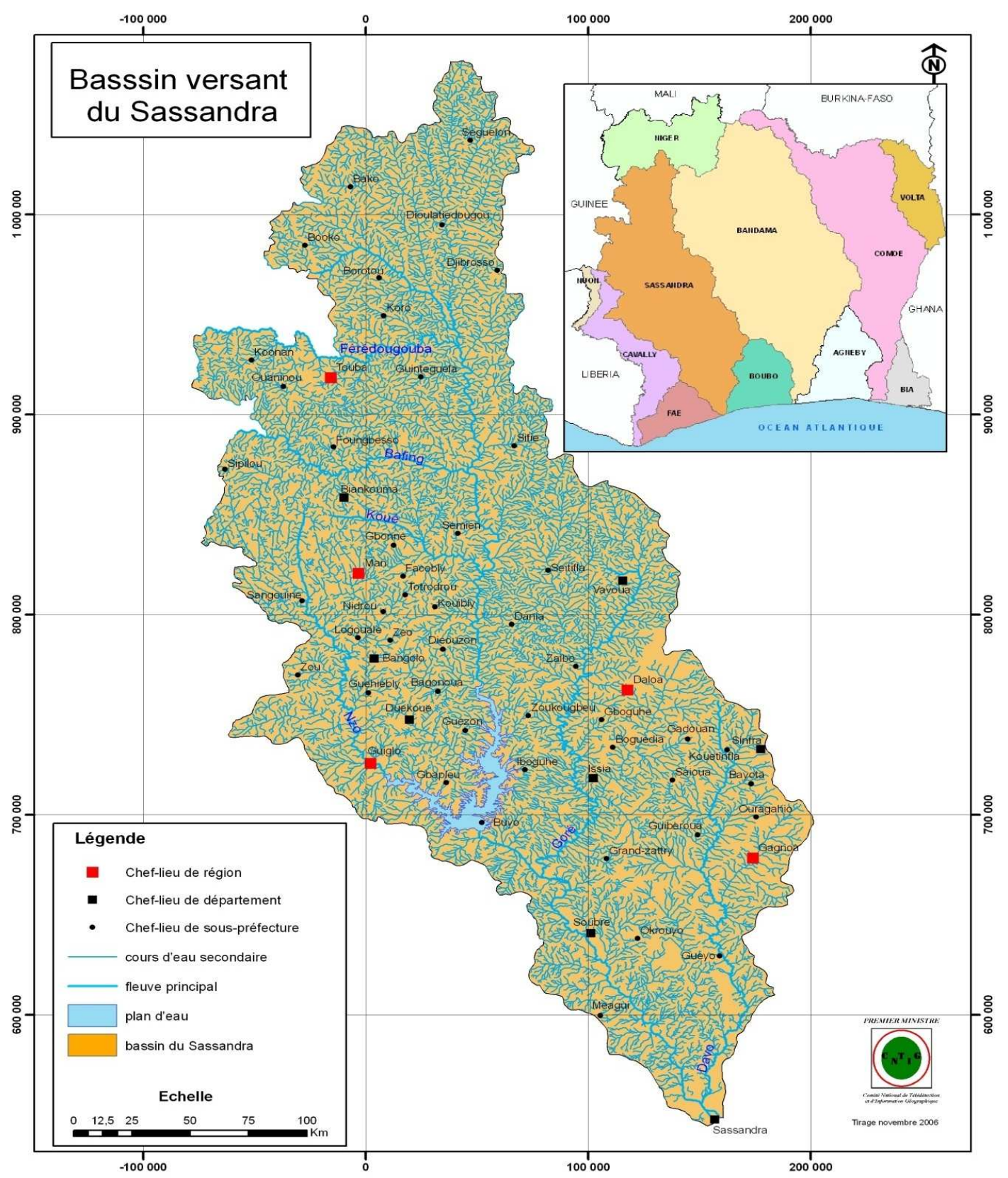

Figure 1: Localisation du bassin versant du fleuve Sassandra (Côte d'Ivoire). 
Tableau 1: Valeurs moyennes de QMO et Ip animaux (Villaud et Varagnat, 1983).

\begin{tabular}{lcccc}
\hline & $\begin{array}{c}\text { Bovins } \\
(\mathbf{2 5 0 - 4 0 0} \mathbf{~ k g})\end{array}$ & $\begin{array}{c}\text { Ovins } \\
\mathbf{( 4 5} \mathbf{~ k g})\end{array}$ & $\begin{array}{c}\text { Caprins } \\
\mathbf{( 4 5} \mathbf{~ k g})\end{array}$ & $\begin{array}{c}\text { Porcins } \\
\mathbf{( 3 0 - 8 0} \mathbf{~ k g})\end{array}$ \\
\hline QMO (kg/tête) & 2 & 0,6 & 0,6 & 0,32 \\
$\mathrm{Ip}\left(\mathrm{m}^{3} / \mathrm{kg}\right.$ de MO) & 0,38 & 0,3 & 0,3 & 0,56 \\
\hline
\end{tabular}

Tableau 2 : Valeurs des coefficients Cres, Cms et Ip (Billaud et Varagnat, 1983; FAO, 1998).

\begin{tabular}{ccccc}
\hline \multirow{2}{*}{ Typologie des résidus } & \multicolumn{3}{c}{ Sources } \\
\cline { 3 - 5 } Cultures & Type de résidu & Cres & Cms & Ip ( $\mathbf{m}^{3} / \mathbf{t}$ de MS $)$ \\
\hline \multirow{2}{*}{ Riz } & Paille & 1,757 & 0,87 & 360 \\
& Balle & 0,267 & 0,98 & 300 \\
\hline \multirow{2}{*}{ Maïs } & Rafle & 0,20 & 0,89 & 300 \\
& Epis & 0,273 & 0,93 & 300 \\
& Tige & 2,00 & 0,85 & 295 \\
Palmier à Huile & Grappe & 0,23 & 0,50 & 300 \\
& Fibre & 0,14 & 0,60 & 300 \\
\hline Cacao & Coque & 0,065 & 0,90 & 300 \\
Café & Cabosse & 1,00 & 0,85 & 300 \\
Coton & Coque & 2,10 & 0,85 & 300 \\
Manioc & Tige & 2,755 & 0,88 & 300 \\
Mil & Tige & 0,062 & 0,85 & 300 \\
& Tige & 1,750 & 0,85 & 278 \\
\hline
\end{tabular}

\section{RESULTATS}

La Figure 2 présente le volume de biogaz estimé à partir des déjections humaines dans les différentes localités du BVS. Le volume maximum de biogaz est obtenu à Soubré $\left(4,5 \times 10^{6} \mathrm{~m}^{3}\right)$. Le département de
Daloa vient en seconde position avec $2 \times 10^{6}$ $\mathrm{m}^{3}$ de gaz produit. Les départements de Gagnoa, Vavoua, Man et Issia ont sensiblement le même potentiel de biogaz produit (environ $1,7 \times 10^{6} \mathrm{~m}^{3}$ ). Quant aux localités de Djibrosso, Sifié, bako, 
Dioulatiédougou et Seguelon, elles sont caractérisées par de très faibles volumes de biogaz produit.

Le volume de biogaz issu des résidus d'élevage des différentes localités du BVS est illustré par la Figure 3. Les départements de Soubré, Touba et Daloa renferment les productions totales les plus élevées $\left(6 \times 10^{6}\right.$ à $6,8 \times 10^{6} \mathrm{~m}^{3}$ ) de biogaz. Ensuite, viennent les départements de Daloa et Sassandra avec respectivement $5,9 \times 10^{6}$ et $5,2 \times 10^{6} \mathrm{~m}^{3}$. C'est à Bangolo et Sinfra que les plus faibles productions de biogaz ont été enregistrées (moins de $10^{6} \mathrm{~m}^{3}$ ). La contribution des déjections dans la production de biogaz, d'une part de l'ensemble des animaux et d'autre part de chaque type d'élevage, diffère d'une région à l'autre. En considérant les départements à grande productivité de biogaz, cette contribution est de $83 \%$ dans le département de Touba contre $46 \%$ à Daloa et $14 \%$ à Soubré. Au regard du type d'élevage, le biogaz issu des déjections de bovins domine la production en biogaz dans les départements de Daloa, Biankouma, Sassandra et Touba tandis qu'à Issia, Vavoua et Soubré, la production de biogaz est dominée par la contribution des déjections de caprins. A l'exception de Soubré, la contribution des déjections de porcins dans la production de biogaz est relativement très faible dans les différentes localités du BVS (moins de 10\%).

Les volumes de biogaz obtenus à partir des résidus agricoles sont répartis en deux catégories : ceux issus de résidus de cultures de rente et ceux provenant des résidus de cultures vivrières. La Figure 4 présente la variation du volume de biogaz des résidus de chaque type de culture de rente exercé dans le BVS. L'on constate que le potentiel de biogaz des cultures de rente est dominé par les résidus des cultures de café et de cacao. A Daloa, Issia et Duekoué, ce potentiel est dominé par les résidus de café tandis qu'à Soubré et à Gagnoa, il est essentiellement dû aux résidus de cacao. Cependant, à Vavoua et Touba, le volume de biogaz résultant des résidus de coton est dominant, avec des proportions respectives de $89,6 \%$ et $61 \%$ du volume total de biogaz desdites localités. Le volume de biogaz enregistré dans le BVS est plus élevé dans le département de Soubré (78 $\mathrm{x} 10^{6} \mathrm{~m}^{3}$ ) qui est suivi respectivement des départements de Daloa $\left(65 \times 10^{6} \mathrm{~m}^{3}\right)$, Issia (64 x $\left.10^{6} \mathrm{~m}^{3}\right)$, Gagnoa $\left(42 \times 10^{6} \mathrm{~m}^{3}\right)$ et Duekoué $\left(42 \times 10^{6} \mathrm{~m}^{3}\right)$. Dans les autres localités du BVS, le volume de biogaz résultant des résidus de cultures de rente reste faible $(6 \mathrm{x}$ $10^{6}$ à $15 \times 10^{6} \mathrm{~m}^{3}$ ).

Concernant les cultures vivrières, la Figure 5 montre les variations du volume de biogaz issu des résidus des différentes cultures vivrières pratiquées dans le BVS. De façon générale, on note une forte contribution des résidus de riz et de maïs $(24-90 \%)$ dans la production de gaz dans la région. Le volume maximal de biogaz a été obtenu à Vavaoua $\left(37,9 \times 10^{6} \mathrm{~m}^{3}\right)$ tandis le volume minimal a été enregistré à Sinfra $\left(2,4 \quad x \quad 10^{6} \mathrm{~m}^{3}\right)$. A l'exception de Vavoua où la contribution des résidus de maïs dans la production totale de biogaz est plus importante $(74,7 \%)$, dans les autres localités du BVS, cette production est dominée par la contribution des résidus de riz. La contribution des résidus générés par la culture du mil n'est constatée qu'à Man et Biankouma. Toutefois, cette contribution est de très faible proportion $(<1 \%)$.

La Figure 6 présente la variation du volume potentiel de biogaz estimé à partir de l'ensemble des résidus agricoles du BVS. On observe une forte proportion de biogaz résultant des déchets des cultures de rente (62-90\%) dans les localités de Daloa, Issia, Gagnoa, Duékoué, Soubré et Sinfra. Cependant, à Vavoua, Man, Bangolo, 
Biankouma, Guiglo et Touba, la tendance est inversée. Dans ces localités, la contribution des résidus générés par les cultures vivrières domine la production de biogaz (65 - 93,5\%).

La Figure 7 présente le volume total de biogaz produit par les différents types de résidus rencontrés dans les localités du BVS. Le volume total de biogaz dans chaque localité est considérablement influencé par le potentiel de biogaz des résidus agricoles. Les excréments humains dans toutes les localités ont une faible proportion dans ce volume de gaz. C'est le département de Soubré qui renferme le potentiel de biogaz le plus important. Par contre, les localités de Djibrosso, Sifié, Bako, Dioulatiedougou et Seguelon ont les potentiels de biogaz les plus faibles.

Les modes d'utilisation actuelle des différents résidus dans le BVS sont présentés dans le Tableau 3. Les déchets sont essentiellement incinérés à l'air libre, à l'exception des excréments humains. On note

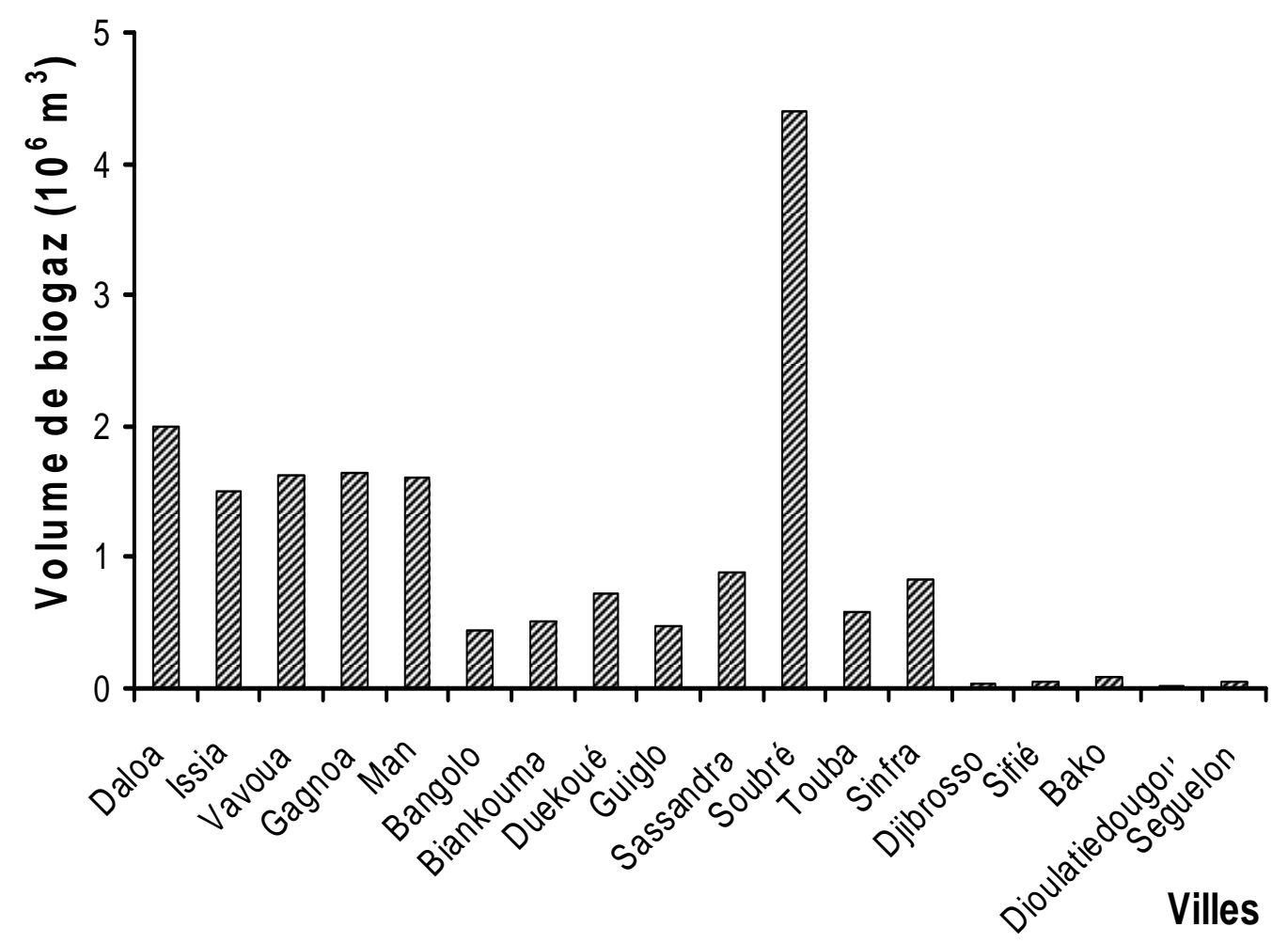

Figure 2: Volume annuel de biogaz produit à partir des excréments humains dans les différentes localités du bassin versant du fleuve Sassandra. 


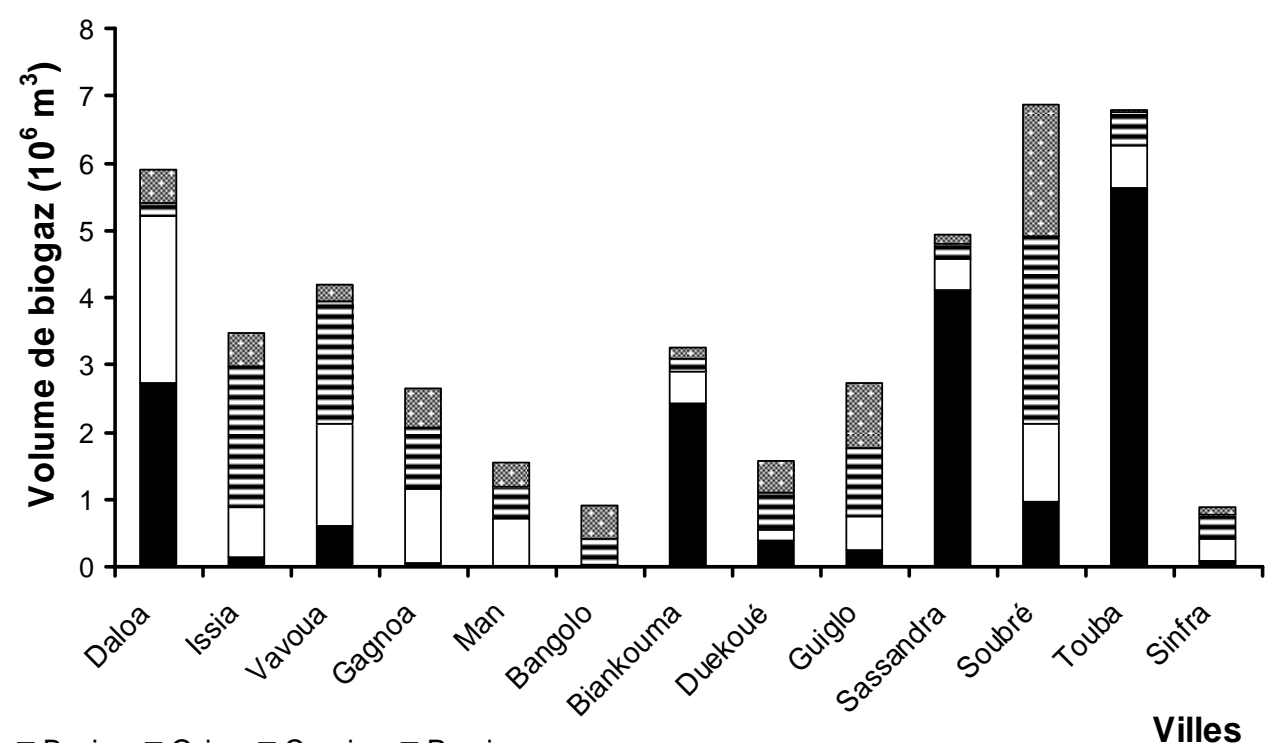

- Bovins $\square$ Ovins $\boxminus$ Caprins Porcins

Villes

Figure 3: Volume annuel de biogaz produit à partir des déjections animales dans les localités du bassin versant du fleuve Sassandra.

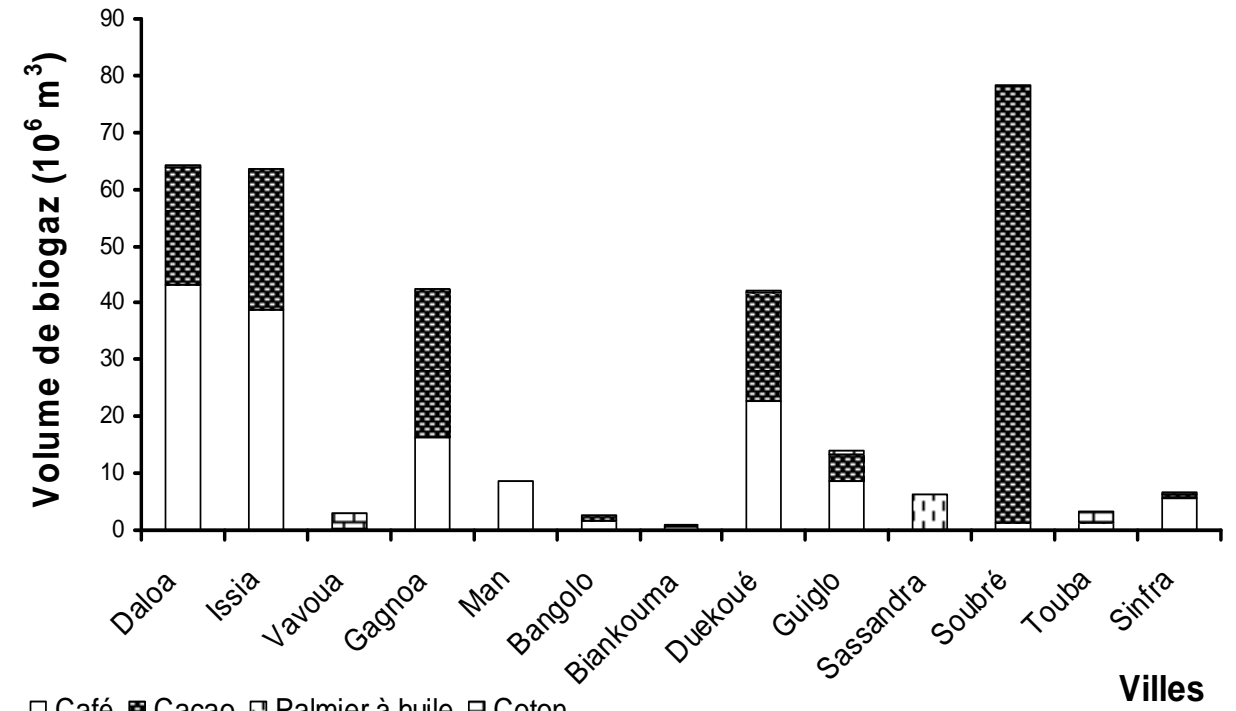

Figure 4: Volume annuel de biogaz produit à partir des résidus des cultures de rente dans les localités du bassin versant du fleuve Sassandra. 


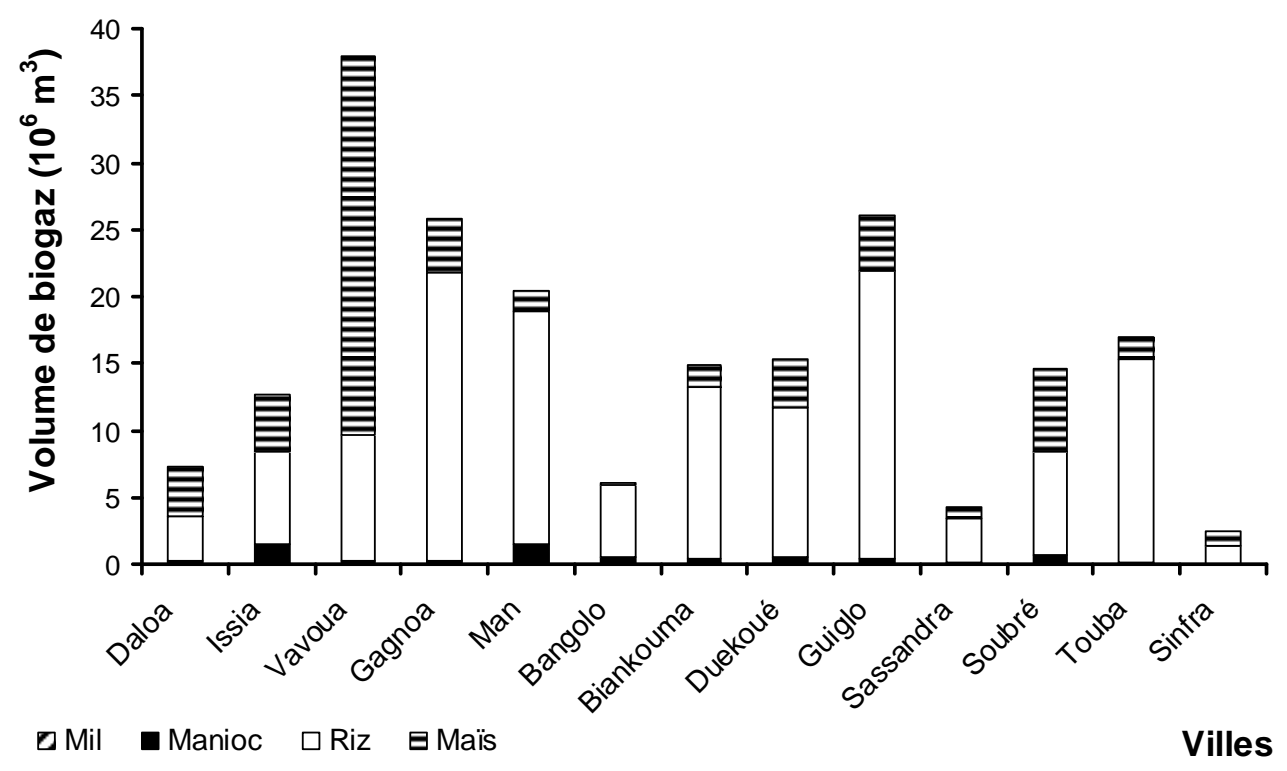

Figure 5: Volume annuel de biogaz produit à partir des résidus de cultures vivrières dans les localités du bassin versant du fleuve Sassandra.

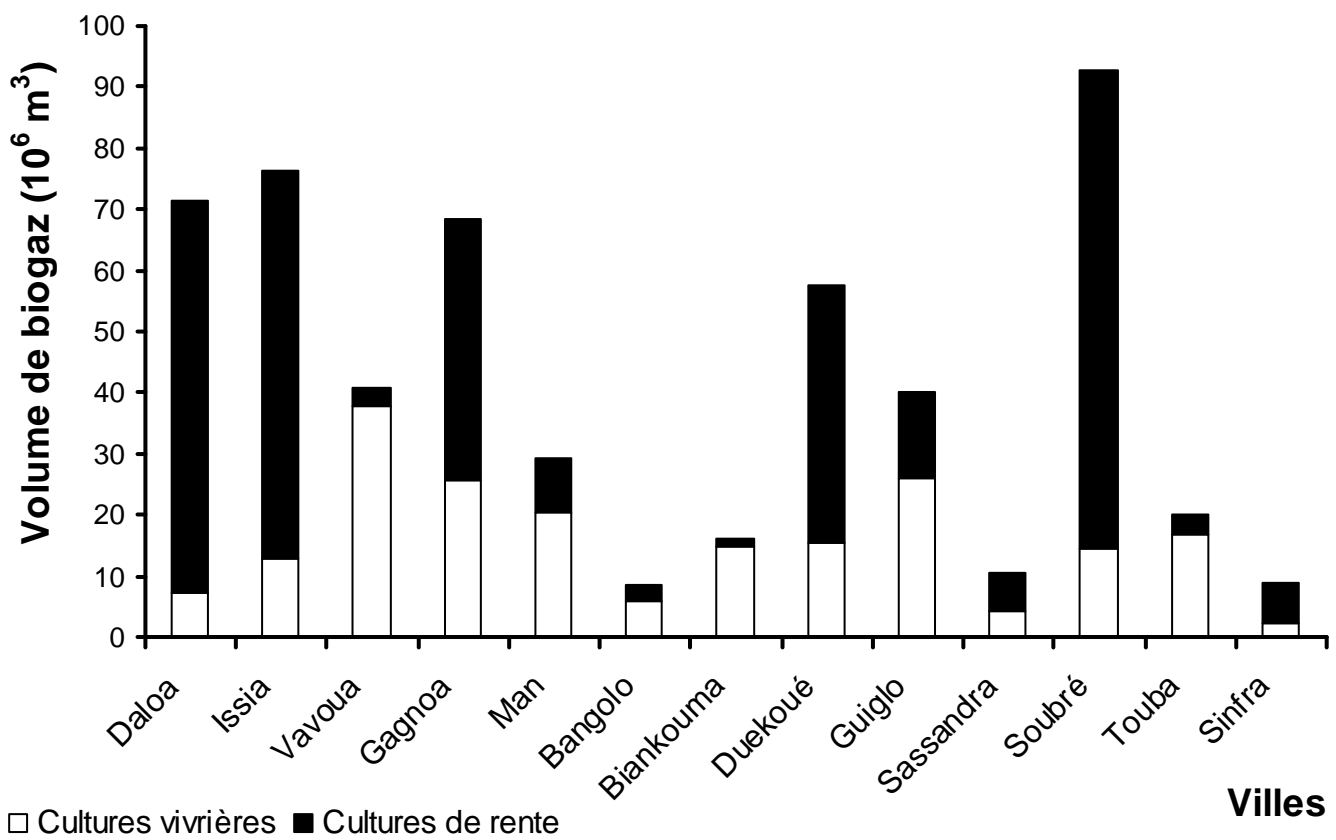

Figure 6: Volume annuel de biogaz estimé à partir des résidus agricoles dans les différentes localités du bassin versant du fleuve Sassandra. 


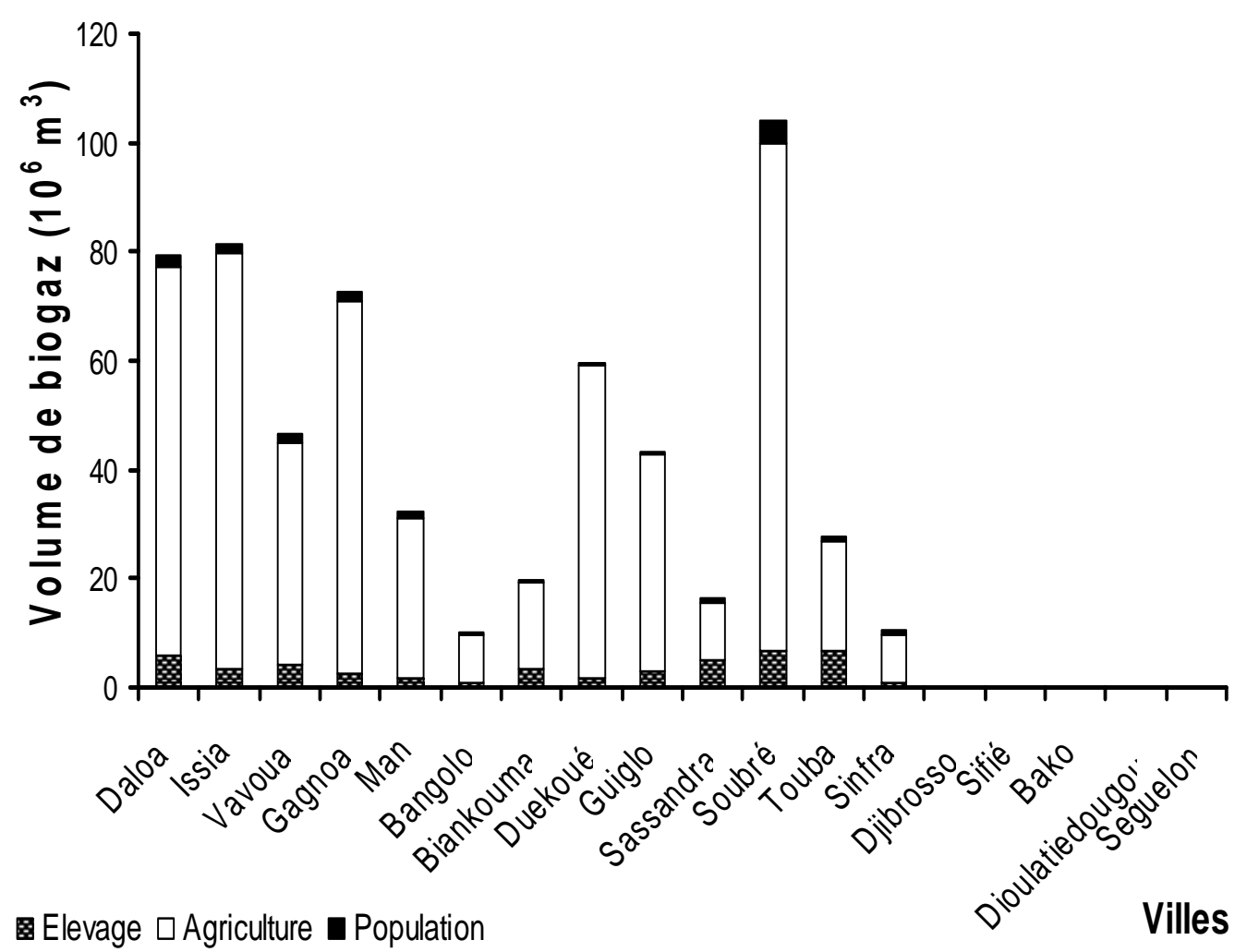

Figure 7: Volume annuel globale de biogaz estimé dans les localités du bassin versant du fleuve Sassandra.

aussi l'utilisation d'une fraction des déchets pour satisfaire les besoins énergétiques domestiques. La seule valorisation industrielle observée est celle des coques de palmes dans la production de vapeur industrielle. Les tiges des cultures vivrières sont essentiellement compostées sur les parcelles de culture. Cette pratique participe à l'amélioration de la qualité du sol, notamment dans l'augmentation de la teneur d'humus. Par ailleurs, il y a une dizaine de petites unités de production de biogaz implantées sur des fermes. S'agissant des excréments humains, ils sont soit stockés dans des fosses septiques et/ou latrines plus ou moins améliorées, soit rejetés dans la nature. Aucune production de biogaz au niveau domestique n'a pu être observée dans le BVS. Pour les différents résidus agricoles, il n'existe pas de statistique sur leurs modes de valorisation. Toutefois, la masse de déchets agricoles incinérés dans l'air libre paraît à l'observation la plus importante. 
Tableau 3: Modes d'utilisation actuelle des différents résidus rencontrés dans le bassin versant du fleuve Sassandra.

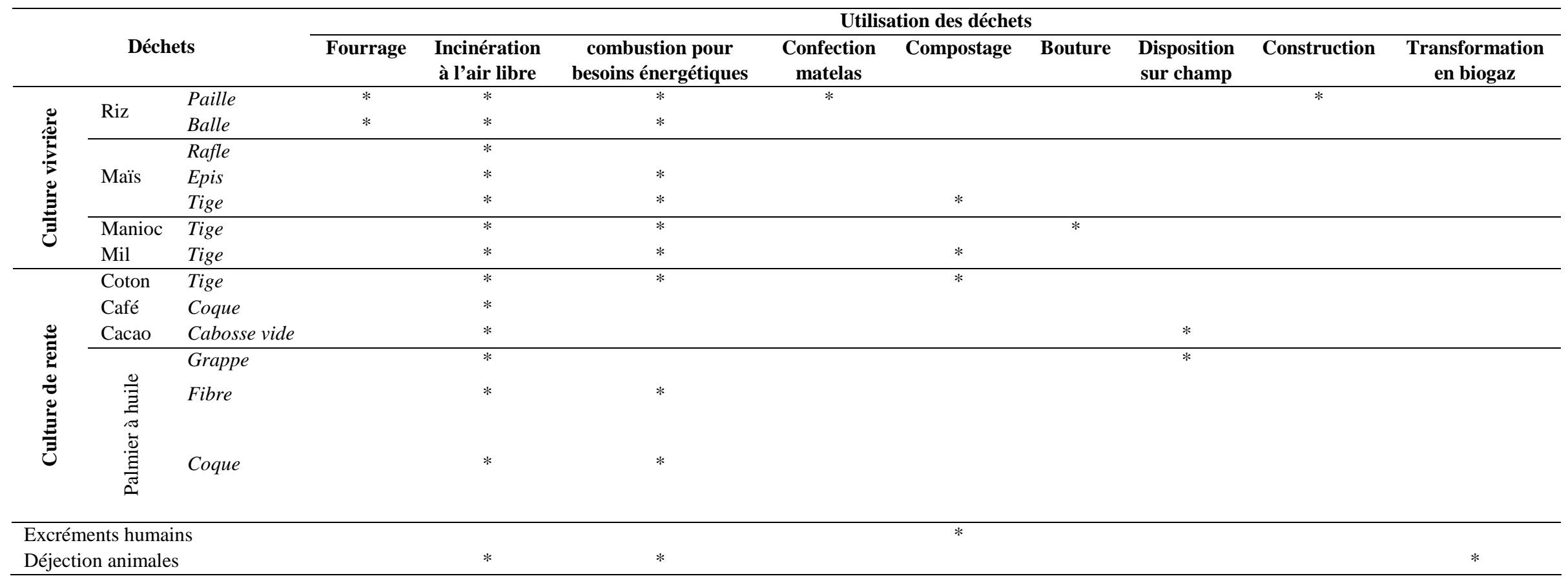




\section{DISCUSSION}

Le potentiel en biogaz des excréments humains de Soubré est le plus important dans le BVS. Ce résultat peut s'expliquer par la forte densité de population dans cette localité. Cette forte densité de population s'expliquerait par la migration des individus dans le département de Soubré pour les activités agricoles, notamment la culture de café et de cacao (Boutillier, 1971; Léonard et Vimard, 2005).

Le potentiel élevé en biogaz des déjections d'élevage à Touba est dû à la contribution importante des déchets de bovins qui est de $82 \%$ du volume total de biogaz émanant des déjections animales. Cette situation pourrait s'expliquer par le fait que le département de Tabou est localisé dans le nord de la Côte d'Ivoire qui est une zone agroécologique favorable à l'élevage du bétail en général et des bovins en particulier (Ndabalishye, 1995 ; FAO, 2003). Concernant les cultures vivrières, la présence des résidus de riz dans tous les départements est vraisemblablement due au fait que cette partie du territoire ivoirien offre des conditions pédoclimatiques favorables à sa culture (Berger, 1988). Le volume total de biogaz estimé à partir des résidus du riz est de 136,9 x $10^{6} \mathrm{~m}^{3}$. Pour les cultures de rente, le potentiel de biogaz repose essentiellement sur les cultures de café et de cacao. Ce résultat serait lié à la politique agricole de la Côte d'Ivoire qui encourage la culture de ces plantes et au fait que le BVS est une zone agro-écologique favorable à leur culture. Le BVS est d'ailleurs aujourd'hui la nouvelle zone de culture intensive du café et de cacao en Côte d'Ivoire. La production maximale de biogaz estimée dans le département de Soubré serait donc influencée par une importance contribution des résidus des cultures de rente, notamment le café et le cacao. En somme, les résultats obtenus montrent l'importance des résidus agricoles comme substrat de production de biogaz.

L'absence d'unité de production de biogaz dans le BVS, alors que les potentialités en substrats organiques pour la production de ce type d'énergie dans ledit bassin sont énormes, pourrait s'expliquer par l'absence de politique de vulgarisation et de dissémination d'unité de production de biogaz dans le milieu rural tout comme au Népal, en Chine et en Inde (USAID, 2007). Les disparités observées dans la répartition des substrats (déchets d'élevage, résidus agricoles et excréments humains) dans les différentes localités du BVS seraient dues à l'inégale répartition des populations ainsi que des surfaces culturales. L'inégale répartition des surfaces culturales pourrait s'expliquer par le fait que les exploitations n'ont pas la même taille en fonction de la spéculation et les cultures sont établies dans différentes zones agroécologiques dans le BVS. Cette situation pourrait aider à orienter les choix technologiques et la méthodologie d'implantation d'unités de biogaz dans le BVS. En effet, l'utilisation des excréments humains pour la production de biogaz, va nécessiter une sensibilisation des populations sur l'utilisation de ce substrat en raison des tabous (Duncker et al., 2007). L'exploitation de ce substrat en milieu rural, en plus des autres substrats organiques (déjections animales et résidus végétaux), pourrait se faire dans de petites unités de biogaz pour satisfaire les besoins énergétiques domestiques. Dans les grandes localités comme Soubré, Daloa et Gagnoa, l'exploitation des excréments humains ne pourrait se faire que dans de grandes unités de production de biogaz vers lesquelles seront convoyées les boues de vidange des fosses septiques. Le potentiel en biogaz des déjections animales est aussi inégalement réparti sur l'ensemble du BVS. Les localités de Soubré, Touba et Daloa qui renferment les volumes les plus élevés, pourraient bénéficier d'unités semiindustrielles de production de biogaz pour valoriser ces déchets. Cependant, il faudra tenir compte du coût de transport desdits déchets en vue de bien évaluer la rentabilité économique de la production. Par ailleurs, l'installation de petites unités de capacité de 6 $\mathrm{m}^{3}$ sur les sites d'élevage du BVS permettrait de résoudre, du moins partiellement, les besoins énergétiques du milieu rural. Cette approche a été employée avec succès au Népal, en Chine et en Inde (USAID, 2007). 
Les résultats obtenus montrent que les déchets agricoles renfermaient un volume important de biogaz. Cependant, la valorisation desdits déchets en biogaz est une technologie relativement complexe qui nécessite un broyage avant la digestion. Par ailleurs, l'acidification des digesteurs rend complexe l'utilisation de ces substrats par des personnes non outillées. Ces substrats ne sont donc pas à conseiller pour la production domestique de biogaz. Toutefois, ils pourraient être valorisés dans des unités industrielles de fermentation de déchets solides (Gunaseelan, 2004; Chanakya et al., 1999) dans les localités de Soubré, Issia, Daloa et Gagnoa.

\section{Conclusion}

Le potentiel de production de biogaz à partir des déjections humaines et animales ainsi que des déchets agricoles du BVS est considérable. Les résidus agricoles ont un potentiel de production plus important, notamment ceux des cultures de café et de cacao. Les localités de Touba, Soubré et Daloa renferment les potentialités les plus élevées. L'installation d'unités décentralisées de production du biogaz à partir des déchets d'animaux est conseillée. Cependant, pour l'utilisation de résidus agricoles, des unités industrielles peuvent être implantées dans les localités, de Soubré, Daloa, Issia et Gagnoa où ces résidus sont les plus importants. Le gisement de biogaz des déchets agropastoraux et des déjections humaines peuvent permettre de procurer une quantité importante d'énergie. L'exploitation de cette source d'énergie permettrait de préserver d'importantes superficies de forêt et alléger la souffrance des femmes à la recherche de biomasse.

\section{REFERENCES}

Berger L. 1988. L'étude de la filière riz. Rapport final: Présidence de la République de Côte d'Ivoire, Direction et contrôle des grands travaux, 25p.

Billaud V, Varagnat F. 1983. Les dossiers du biogaz, Dossier A: La fermentation méthanique. Cellule Biométhane du GERES, Paris, 72p.
Boutillier JL. 1971. Croissance démographique et croissance économique en Côte d'Ivoire. Cahier O.R.S.T.O.M., Série Science Humaine, 8: 73-79.

Chanakya HN, Srikumar KG, Anand V, Modak J, Jagadish KS. 1999. Fermentation properties of Agro-residues, leaf biomass and urban market garbage in a solid phase biogas fermenter. J. Biomass and Bioenergy, 16:417-429.

Chanakya HN, Rajabapaiah P, Modak JM. 2004. Evolving biomass-based biogas plants: The ASTRA experience. Current Science, 87: 917-925.

Cho JG, Park SC, Chang HN. 1995. Biochemical methane potential and solid state anaerobic digestion of Korean food wastes. Bioressource Technology, 22: 245-253.

Chynoweth DP, Owens JM, Legrand R. 2001. Renewable methane from anaerobic digestion of biomass. Renewable Energy, 22: 1-8.

Duncker LC, Matsebe GN, Moilwa N. 2007. The social/cultural acceptability of using human excreta (faeces and urine) for food production in rural settlements in South Africa. Report to the Water Research Commission No TT 310/07.

ESMAP. 2005. Atelier sur l'énergie rurale pour un développement durable en milieu rural en Côte d'Ivoire. World Bank Energy Sector Management Assistance Programme, Golf Hotel, Abidjan, 30-31 Janvier 2002.

FAO. 1998. Proceedings of the regional expert consultation on modern application of biomass energy. FAO Regional Wood Energy Development programme in Asia, Report 36, Bangkok, 22p.

FAO. 2003. Cattle and small ruminant production system in sub-Saharan Africa: a systematic review. Food and Agricultural Organisation, Rome.

FAO. 2005. Global livestock production and health atlas. Food and Agricultural Organisation. Rome.

Girard G, Sircoulon J, Touchebeuf P. 1971. Aperçu sur le régime hydrologique. In Le Milieu Naturel de la Côte d'Ivoire, Avenard JM, Girard M, Sircoulon J, 
Touchebeuf P, Guillaumet JL, Adjdnohoun E, Perraud A (éds). ORSTOM: Paris; 109-155.

Gowga MC, Raghavan GSV, Ranganna B, Barrington S. 1995. Rural waste management in a south Indian village: A case study. Bioresource Technology, 53: 147-164.

Gunaseelan VN. 2004. Biochemical methane potential of fruits and vegetable solid waste feedstocks. Biomass and Bioenergy, 26: $389-399$.

IEA. 1998. World Energy Outlook, International Energy Agency, 1998. IEA, Paris.

IEA. 2000. World Energy Outlook, International Energy Agency, 2000. IEA, Paris.

IEA. 2002. World Energy Outlook, International Energy Agency, 2002. IEA, Paris.

INS. 2000. Statistique de la population de la Côte d'Ivoire. Institut National de la Statistique.

Kivaisi AK, Rubindamayugi MS. 1996. The potential of agro-industrial residues for production of biogas and electricity in Tanzania. Wrec; 917-921.

Kumar A, Purohit P, Rana S, Kandpal TC. 2002. An approach to the estimation of the value of agricultural residues used as biofuels. Biomass and Bioenergy, 22: 195-203.

Léonard E, Vimard P. 2005.Crises et Recompositions d'une Agriculture Pionnière en Côte d'Ivoire: Dynamiques Démographiques et Changements Economiques dans le Bas-Sassandra (Côte d'Ivoire). Collection Hommes et Sociétés : Paris, IRD-Karthala; 368 p.

Mehretu A, Mutambira C. 1992. Gender differences in time and energy costs of distance for regular domestic chores in rural Zimbabwe: a case study of the Chiduku Communal Area. World Development, 20: 1675-1683.

Moller HB, Sommer SG, Ahring BK. 2004. Methane productivity of manure, straw and solid fraction of manure. Biomass and Bioenergy, 26: 485-495.
Nabinta RT, Yahaya MK, Olajide BR. 2007. Socio-economic implications of rural energy exploitation and utilisation on sustainable development in Gombe State, Nigeria. J. Soc. Sci.,15: 205-211.

Nagamani B, Ramasamy K. 2005. Biogas production technology: an Indian perspective. Tamil Nadu Agricultural University of India, 18p.

Ndabalishye I. 1995. Agriculture vivrière Ouest-Africaine à travers le cas de la Côte d'Ivoire. Institut des Savanes, 383p.

Nelson RG, Walsh M., Sheehan JJ., Graham R. 2004. Methodology for estimating removable quantities of agricultural residues for bioenergy and bioproduct use. Applied Biochemistry and Biotechnology, 113: 13-26.

OMS. 2000. Addressing the Links between Indoor Air Pollution, Household Energy and Human Health. Based on the WHOUSAID global consultation on the health impact of indoor air pollution and household energy in developing countries (Meeting report). Washington DC 3-4 May.

PNUD. 2001. Biogas technology in agricultural regions, Tanzania. Project: Promotion of Low Cost Biogas Technology to Resource Poor Farmers in Tanzania, $3 \mathrm{p}$.

PNUD. 2003. Human Development Report, United Nations Development Programme, New York.

USAID. 2007. Biogas: Retrospect and prospect, Georgia, Rural Energy Program, $43 \mathrm{p}$.

Wauthelet M. 2001. La biométhanisation des déchets solides agricoles. Thèse de Doctorat, Université Catholique de Louvain, 58p.

Zhang R, Zhang Z. 1999. Biogasification of rice straw with an anaerobic-phased digester system. Bioresource Technology, 68: $235-245$. 